\title{
Depurated Inversion Method for Orbital-Specific Exchange Potentials
}

\author{
A.M.P. Mendez, D.M. Mitnik, J.E. Miraglia ${ }^{\dagger}$
}

April 18, 2022

\begin{abstract}
This work presents exchange potentials for specific orbitals calculated by inverting Hartree-Fock wavefunctions. This was achieved by using a Depurated Inversion Method. The basic idea of the method relies upon the substitution of Hartree-Fock orbitals and eigenvalues into the Kohn-Sham equation. Through inversion, the corresponding effective potentials were obtained. Further treatment of the inverted potential should be carried on. The depuration is a careful optimization which eliminates the poles and also ensures the fullfilment of the appropriate boundary conditions. The procedure developed here is not restricted to the ground state or to a nodeless orbital and is applicable to all kinds of atoms. As an example, exchange potentials for noble gases and term-dependent orbitals of the lower configuration of Nitrogen are calculated. The method allows to reproduce the input energies and wavefunctions with a remarkable degree of accuracy.
\end{abstract}

*alemendez@iafe.uba.ar

†Instituto de Astronomía y Física del Espacio, CONICET-UBA, Buenos Aires, Argentina. 


\section{INTRODUCTION}

The successful idea of replacing a many-body, non-local interaction by an effective oneelectron equation opened up the possibility of studying extremely complex systems with high accuracy. In this context, the success of the Kohn-Sham density-functional theory $\underline{1,2}$ (DFT) began when crucial developments in its exchange-correlation terms gave the theory predictive power to compete with well-developed wavefunction methods ${ }^{\underline{3}}$. The importance of the exchange-correlation potentials in chemical physics has been emphasized by Bartlett $+\underline{4.5}$. Exchange potentials are in general constructed by local approximations to the nonlocal Hartree-Fock exchange operator (i.e. the Slater potential ${ }^{6}$, the optimized effective potential ${ }^{\underline{\underline{ }} \underline{\underline{9}}}$, the Krieger-Li-Iafrate $\underline{\underline{10}}$ and several others $\underline{\underline{11}} \underline{\underline{14}}$ ).

The atomic collision community, on the other hand, is also eager to accurately determine effective one-electron local potentials which would allow to generate in a simpler way the wavefunctions of the particles interacting in a scattering process. In particular, we need to represent an orthonormal set of bound and continuum states to calculate the transition probabilities. This should include detailed $n l$-orbital potentials, a feature missing in most of the standard density functional methods. Soft pseudopotentials like ABINIT $\underline{15}$ or USPP $\underline{16}$ cannot be used because they overlook the information of the internal region of the wavefunctions. The features of this region can play a very important role, such as the cusp conditions in the processes of electron capture and ionization. In an attempt to meet the needs of both chemist and collisionist communities, we strove to obtain accurate and simple specific $n l$-orbital local potentials.

How to determine central potentials from known electron wavefunctions and densities is a well studied subject in the DFT community $\underline{\underline{17}} \underline{\underline{19}}$. The extraction of the true Kohn-Sham exchange-correlation potential from near-exact electronic densities has been demonstrated, with particular reference to two-electron systems like $\mathrm{He}^{20}$, He-isoelectronic ions ${ }^{21}$, and $\mathrm{H}_{2} \underline{\underline{20}, 22}$ as well as exact soluble models (for example, an external harmonic potential as in Filippi et $a l \underline{\underline{23}}$ ).

Some other works start with a particular Kohn-Sham potential and solve the corresponding equations, obtaining the KS orbitals $\underline{24} \underline{-26}$. Through inversion, they obtain a reconstructed KS potential, which agrees almost everywhere with the original one, except in some regions where huge oscillations arise. In some cases, the reconstructed potential may be distorted beyond recognition $\underline{20,27}$. The same type of procedure was suggested many years ago by Hilton et al., in applications circumscribed to the calculation of photoionization processes of atoms $\underline{28,29}$, water $\underline{30}$ and other molecules $\frac{31}{1,32}$. These papers, in turn, refer to the earlier work in atomic polarizability carried out by Sternheimer $\underline{\underline{33}}$ and Dalgarno and Parkinson $\underline{34}$. However, they focused on the final photoionization cross section results, and did not provide details about the quality of the potentials and the wavefunctions they generated.

Assuming the validity of the separation between exchange and correlation functionals, we will focus here only on the calculation of the exchange contribution to the potential. Since Hartree-Fock does not include the correlations, our approach allows to obtain the "exact" one-electron local potential representing the exchange interactions. Strictly speaking, the method does not rely on the KS inversion formula since the Hartree-Fock solutions were the ones used for the inversion. That is, we solved a KS-type equation, but rather than having KS-orbitals, we operated directly with the Hartree-Fock wavefunctions. For open-shell 
atoms, we were able to find orbital spin-polarized exchange potentials, this being crucial, for instance, to find the hyperfine coupling constants 35,36 .

However, this is not a simple task, and probably that is why the method presented here has not been widely applied in the past. If the wavefunction has nodes, it will produce huge poles in the potential. Moreover, even for nodeless states, the asymptotic decaying behavior of the bound wavefunctions produces severe numerical difficulties, making the inversion operation intractable sometimes. In our method, a depuration procedure follows the inversion. This depuration implies, first, the annihilation of the poles. Then, a careful optimization of the potential which ensures the fulfillment of the appropriate boundary conditions.

The work is organized as follows. Section 2 describes the method, which includes the inversion procedure (2.1), the potential depuration (2.2) and its further optimization (2.3). Section 3 presents the resulting effective potentials for the orbitals corresponding to the ground states of different noble gases, including a thorough examination of the wavefunctions generated by these potentials (3.1). The corresponding exchange potentials are discussed in (3.2), comparing the potentials for specific $-n l$ orbitals with averaged potentials. Results of the same calculations for the Nitrogen atom are provided in (3.3). Atomic units are used unless otherwise specified.

\section{THEORY}

\subsection{The direct inversion method}

The radial part of the Schrödinger equation for an electron in a local and central potential is

$$
\left[-\frac{1}{2} \frac{d^{2}}{d r^{2}}+\frac{l(l+1)}{2 r^{2}}+V_{n l}(r)\right] u_{n l}(r)=\varepsilon_{n l} u_{n l}(r) .
$$

We assume the following hypothesis: If the wavefunctions $u_{n l}$ are replaced by the solutions of an Hartree-Fock calculation $u_{n l}^{\mathrm{HF}}$, then, the corresponding effective local potentials $V_{n l}^{\mathrm{HF}}$ that generate such wavefunctions should exist. Based on this we converted the HF method into a set of Kohn-Sham equations, whose solutions are the Hartree-Fock wavefunctions:

$$
\left[-\frac{1}{2} \frac{d^{2}}{d r^{2}}+\frac{l(l+1)}{2 r^{2}}+V_{n l}^{\mathrm{HF}}(r)\right] u_{n l}^{\mathrm{HF}}(r)=\varepsilon_{n l}^{\mathrm{HF}} u_{n l}^{\mathrm{HF}}(r) .
$$

The effective potentials given by,

$$
V_{n l}^{\mathrm{HF}}(r)=V^{\mathrm{C}}(r)+V^{\mathrm{dir}}(r)+V_{n l}^{\mathrm{x}}(r),
$$

are composed of the external potential $V^{\mathrm{C}}$ (the Coulomb field of the nucleus), the direct (or Hartree) potential $V^{\text {dir }}$ (the electrostatic electron repulsion), and the orbital exchange potentials $V_{n l}^{\mathrm{x}}$. We have ignored the correlation term since the HF solutions do not include it.

Since the solutions $u_{n l}^{\mathrm{HF}}$ are known (calculated numerically with the HF code by C. F. Fischer ${ }^{37}$, and the NRHF code by W. Johnson ${ }^{38}$ ) we proceeded to directly invert the KohnSham-type equations:

$$
V_{n l}^{\mathrm{HF}}(r)=\frac{1}{2} \frac{1}{u_{n l}^{\mathrm{HF}}(r)} \frac{d^{2}}{d r^{2}} u_{n l}^{\mathrm{HF}}(r)-\frac{l(l+1)}{2 r^{2}}+\varepsilon_{n l}^{\mathrm{HF}},
$$


obtaining the $H F$ inverted potential $V_{n l}^{\mathrm{HF}}(r)$. Assuming a Coulombic-type shape, it is convenient to define an $H F$ inverted charge

$$
Z_{n l}^{\mathrm{HF}}(r) \equiv-r V_{n l}^{\mathrm{HF}}(r) .
$$

The direct computation of (4) is known to pose serious numerical problems ${ }^{20}$. First, the presence of (genuine) nodes in the wave function to be inverted produces poles and unrealistic features around them. This has led to the general consensus that the inversion method can only be used for nodeless orbitals ${ }^{26}$. Second, numerical rounding up of the exponential decay of the bound states hinders the corresponding inverted potential from having the physically desired asymptotic form. Moreover, there is a third problem at the very heart of the Hartree Fock method: the exact solutions may have oscillations (and therefore, spurious nodes) in the large-r or "tail" region of the functions. The existence of these spurious nodes in Hartree Fock was already suggested by Fischer $\stackrel{37}{ }$. This failure is not caused by the numerical scheme but it is inherent to the method. Probably, these nodes are surviving long-range exchange effects due to the non-local character of the Hartree-Fock wavefunctions: the behavior of a particular orbital depends on all others. We have found the same spurious nodes at the same places even using different numerical codes. As a general rule, the spurious nodes appear at very long distances, in regions where the amplitude of the wavefunction is very small. Therefore, their existence has no practical consequences, and they can be ignored in any general Hartree-Fock calculation. However, this is not true as far as the inversion procedure is concerned, as we will discuss in the next section. Other examples where the presence of orbital nodes (both formal and those in the tail region) can be problematic in inversion procedures can be found in the literature (see for instance Peach et al. $\underline{\underline{39}}$ ).

\subsection{The depurated inversion method}

The difficulties mentioned above make it very hard to obtain the correct $V_{n l}^{\mathrm{HF}}(r)$ potentials using the simple inversion formula given by Eq. (4). To overcome these troubles we have developed a depurated inversion method (DIM) which optimizes the effective charges rather than the effective potentials. We managed to constrain any potential to have the right boundary conditions by enforcing the effective depurated inverted charge to behave as follows:

$$
Z_{n l}^{\mathrm{DIM}}(r) \rightarrow \begin{cases}Z_{N} & \text { as } r \rightarrow 0 \\ 1 & \text { as } r \rightarrow \infty\end{cases}
$$

where $Z_{N}$ is the nuclear charge. Once the charge is determined at the boundaries, we can obtain a smooth analytic expression for $Z_{n l}^{\mathrm{DIM}}(r)$, fitting the $Z_{n l}^{\mathrm{HF}}(r)$ for the largest possible range, except in the neighborhood of the nodes. All this can be accomplished by imposing the effective DIM charge to fit the following analytical expression:

$$
Z_{n l}^{\mathrm{DIM}}(r)=\sum_{j} \alpha_{j} e^{-\beta_{j} r}+1
$$

with $\Sigma_{j} \alpha_{j}=Z_{N}-1$.

As a clear instance of the numerical problems mentioned and the way propose to solve them, we show, in Figure 1, the orbital $u_{2 s}^{\mathrm{HF}}(r)$ of the ground state of the Kr atom (part (a)), 


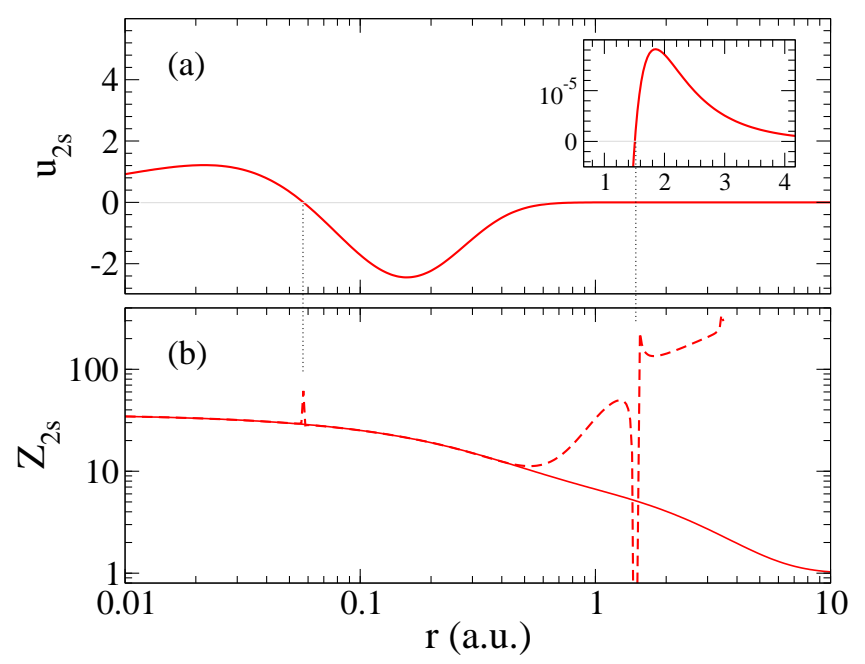

Figure 1: (a) Hartree-Fock orbital $u_{2 s}^{\mathrm{HF}}$ corresponding to the ground state of the Kr atom. It presents two nodes, a genuine one at $r \approx 0.06$ a.u., and a spurious one at 1.51 a.u. (shown in the inset). (b) Dashed line: The corresponding inverted effective charge $Z_{2 s}^{\mathrm{HF}}(r)$, spoiled by the presence of poles. Solid line: Depurated $Z_{2 s}^{\mathrm{DIM}}(r)$ effective charge.

and its correspondent effective charge $Z_{2 s}^{\mathrm{HF}}(r)$ (dashed line curve, in part (b)). First, note that the $2 s$ orbital has a genuine node at $r \approx 0.06$ a.u. which produces the first pole in the effective charge, as shown in the lower graph. The node appears at a relatively low $-r$ value, so the corresponding charge (see Eq. (5) ) is not very sensitive to its presence. Therefore, it is very easy to eliminate the pole from the effective charge (by just erasing a few points around this radius).

All the bound wavefunctions decay exponentially beyond the last turning point $r_{t p}$, defined as the position in which the energy equals the effective potential. At first glance, it seems that the turning point of $u_{2 s}(r)$ is located around $r_{t p} \approx 0.25$, and from that point on, the wavefunction should start to decay exponentially. From the numerically point of view, $r \approx 10 r_{t p}$ is a good point to stop the inversion, since beyond there, the effective charge could begin to diverge. Thus, one might infer that by erasing the points belonging to the neighborhood of the first node, and by stopping the inversion about $10 r_{t p}$, the inversion procedure will work well. However, the dashed curve in Fig. 1(b) shows a completely unphysical $Z_{2 s}^{\mathrm{HF}}(r)$ resulting from the inversion. A very careful examination of the $u_{2 s}^{\mathrm{HF}}(r)$ orbital function evidences the presence of a spurious node at $r=1.51$ a.u., in a region where the amplitude of the wavefunction is less than $10^{-4}$ times the maximum value (see the inset of Fig. 1(a)). Even though this node is completely innocuous for practical matters, it produces devastating effects in the inversion procedure, evidenced by the second huge peak in the $Z_{2 s}^{\mathrm{HF}}(r)$ curve (see Figure 1(b)). This pole is so big that it affects a broad vicinity and causes the abrupt rising of the effective charge for $r>0.5$ a.u.. This is really a surprising result since a priory there is no reason to suspect that a negligible oscillation in the tail of the wavefunction would produce such a big drawback at small distances. Care must be taken then to discard these kind of undesired effects. 


\subsection{Optimization}

The adjustment of the parameters $\alpha_{j}$ and $\beta_{j}$ also requires carefull work. The key issue in the successful approximation is the region chosen for the fitting: it has to be as large as possible, in such a way that $Z_{n l}^{\mathrm{DIM}}(r)$ overlaps the inverted $Z_{n l}^{\mathrm{HF}}(r)$ across a broad range, allowing an accurate fitting procedure, but discarding the points surrounding the nodes. Also, the inversion must be halted at a particular (as large as possible) $r$ value, as soon as the amplitude of the function is too small. Further on, the inversion procedure diverges. Another issue to consider is the self consistency within the computer codes used in the calculations and the particular code used to generate the input wavefunctions. To that end, we make sure that the same specific numerical grid is used, including the derivatives and integrals at the same pivots. The optimization procedure is completed by a number of iteration steps, in which the parameters are optimized to give accurate energies and wavefuntions.

Most density functional approximation methods are based on a variational principle, minimizing the density functionals according to energy (others are defined by density). Without underestimating its importance, energy is only one of the many parameters that characterizes a quantum state. Different trial functions (having different forms) can produce, through a variational procedure, the same final energy. A simple example is given by Bartschat $\underline{40} \underline{41}$ in which two different potentials (one having exchange, the other omitting it) led to producing very similar and accurate energies of the Rydberg series in several quasi-one electron systems. However, a further examination of these potentials shows large discrepancies in scattering calculations $\underline{42}$. Therefore, in addition to the energy criterion, we have included in our optimization method a variational procedure to reproduce accurately the wavefunctions. This is achieved by optimizing the mean values $\langle 1 / r\rangle$ (which characterize the quality of the wavefunction near the origin), and $\langle r\rangle$ (probing it at longer distances). Furthermore, we defined the quantity

$$
\delta=1-\frac{\int u_{n l}^{\mathrm{HF}}(r) u_{n l}^{\mathrm{DIM}}(r) d r}{\int \rho_{n l}^{\mathrm{HF}}(r) d r} .
$$

to determine the accuracy of the orbitals generated by the diagonalization of the DIM potentials and the original HF orbitals.

The effective depurated inversion charge $Z_{2 s}^{\mathrm{DIM}}(r)$ corresponding to the $2 s$ orbital of the $\mathrm{Kr}$ atom resulting from the optimization is shown -solid curve- in Figure1(b). As seen in the figure, both boundary conditions are fulfilled (at the origin, $Z_{2 s} \rightarrow 36$, and asymptotically $Z_{2 s} \rightarrow 1$, as stated in Eq. (맘) $)$.

\section{RESULTS}

\subsection{DIM Potentials, energies and mean values}

The fitting parameters $\alpha_{j}$ and $\beta_{j}$ defining the effective charges $Z_{n l}^{\mathrm{DIM}}(r)$ in Eq. (7) for the noble gases Helium, Neon, Argon and Krypton, are given in Table 1. We have limited the $\alpha_{j}$ and $\beta_{j}$ to six (about two per shell). For Kr, we would probably need two more since there are four shells involved. Having these effective charges, we built the corresponding DIM potentials $V_{n l}^{\operatorname{DIM}}(r)$. By solving the Schrödinger equation (Eq. (1)), we obtained the 
Table 1: Fitting parameters for the effective charge $Z_{n l}^{\mathrm{DIM}}(r)$ for $\mathrm{He}, \mathrm{Ne}$, Ar, and $\mathrm{Kr}$, applying Eq. (7).

\begin{tabular}{|c|c|c|c|c|c|c|c|}
\hline & $n l$ & $\alpha$ & $\beta$ & & $n l$ & $\alpha$ & $\beta$ \\
\hline \multirow[t]{3}{*}{$\mathrm{He}$} & $1 s$ & -0.31745 & 5.04372 & \multirow[t]{3}{*}{$\mathrm{Kr}$} & \multirow[t]{3}{*}{$1 s$} & 5.49263 & 0.884768 \\
\hline & & 1.31745 & 2.50032 & & & 3.94437 & 16.8769 \\
\hline & & - & - & & & 25.5630 & 3.10032 \\
\hline \multirow[t]{9}{*}{$\mathrm{Ne}$} & \multirow[t]{3}{*}{$1 s$} & 7.367687 & 2.417275 & & \multirow[t]{3}{*}{$2 s$} & 9.63120 & 0.575832 \\
\hline & & 1.300360 & 0.126396 & & & 1.84650 & 25.53280 \\
\hline & & 0.331953 & 13.15820 & & & 23.5223 & 4.543350 \\
\hline & \multirow[t]{3}{*}{$2 s$} & 0.297739 & 17.99390 & & \multirow[t]{3}{*}{$2 p$} & 3.20530 & 20.83535 \\
\hline & & 0.668081 & 0.067288 & & & 23.6172 & 3.928520 \\
\hline & & 8.03418 & 2.47221 & & & 8.17750 & 0.636486 \\
\hline & \multirow[t]{3}{*}{$2 p$} & 1.353049 & 8.56948 & & \multirow[t]{3}{*}{$3 s$} & 6.52203 & 0.547357 \\
\hline & & 0.335881 & 0.464942 & & & 24.4475 & 3.657030 \\
\hline & & 7.311070 & 2.090634 & & & 4.03047 & 16.61770 \\
\hline \multirow[t]{15}{*}{$\mathrm{Ar}$} & \multirow[t]{3}{*}{$1 s$} & 6.727570 & 6.177720 & & \multirow[t]{3}{*}{$3 p$} & 23.13135 & 4.010523 \\
\hline & & 4.751090 & 1.343560 & & & 3.325360 & 20.41890 \\
\hline & & 5.521340 & 0.859981 & & & 8.543290 & 0.821218 \\
\hline & \multirow[t]{3}{*}{$2 s$} & 8.90271 & 1.09779 & & \multirow[t]{3}{*}{$3 d$} & 10.05320 & 1.04843 \\
\hline & & 2.36850 & 2.93144 & & & 21.81544 & 4.25746 \\
\hline & & 5.72879 & 6.95913 & & & 3.131360 & 20.6087 \\
\hline & \multirow[t]{3}{*}{$2 p$} & 4.96956 & 6.14455 & & \multirow[t]{3}{*}{$4 s$} & 3.65988 & 0.49000 \\
\hline & & 1.48464 & 10.86843 & & & 26.4565 & 3.17799 \\
\hline & & 10.5458 & 1.30005 & & & 4.88362 & 15.2031 \\
\hline & \multirow[t]{3}{*}{$3 s$} & 10.3202 & 2.33169 & & \multirow[t]{6}{*}{$4 p$} & 7.35713 & 1.00142 \\
\hline & & 4.27115 & 7.33678 & & & 24.2321 & 3.7309 \\
\hline & & 2.40865 & 0.407463 & & & 3.41077 & 22.5680 \\
\hline & \multirow[t]{3}{*}{$3 p$} & 8.43753 & 3.49259 & & & & \\
\hline & & 2.18200 & 10.8595 & & & & \\
\hline & & 6.38047 & 1.07080 & & & & \\
\hline
\end{tabular}


solutions $u_{n l}^{\mathrm{DIM}}(r)$ and the corresponding energies $\varepsilon_{n l}^{\mathrm{DIM}}$. The comparison between the results obtained from the diagonalization of the Hamiltonian with the $V_{n l}^{\mathrm{DIM}}(r)$ effective potential and the original Hartree-Fock orbitals are presented in Table 2, It is remarkable that with such simple analytical expressions for the potentials we were able to reproduce exactly the same energies as the HF method. The only exception is the $4 p$ orbital of $\mathrm{Kr}$, in which both calculations agree up to the fifth significant figure. The fitting procedure also allows to reproduce the original HF wavefunctions with an outstanding degree of accuracy. The agreement between the HF orbitals $u_{n l}^{\mathrm{HF}}(r)$ and the solutions $u_{n l}^{\mathrm{DIM}}(r)$ can be tested through the comparison of the mean values $\langle r\rangle$ and $\langle 1 / r\rangle$, and the computation of quantity $\delta$ defined by Eq. (8). The mean values agree in about $0.1 \%$ while the values of $\delta$ are about $10^{-5}$.

Finally, we calculated the total energy for the ground state of each atom, by using the following expression:

$$
E^{\mathrm{DIM}}=\sum_{n l}\left[\varepsilon_{n l}^{\mathrm{DIM}}-\frac{1}{2} \int \rho_{n l}^{\mathrm{DIM}}(r)\left(V_{n l}^{\mathrm{DIM}}(r)+\frac{Z_{N}}{r}\right) d r\right]
$$

where the density $\rho_{n l}^{\text {DIM }}(r)=\left|u_{n l}^{\text {DIM }}(r)\right|^{2}$. The calculated energies $E^{\text {DIM }}$ are given in Table 2 , together with the total energies obtained by the Hartree-Fock calculations. The comparison shows a notable agreement between both calculations, at about $0.02 \%$.

\subsection{The exchange potential}

Orbital-specific exchange potentials can be obtained accurately by computing the non-local Fock exchange operator. A first local approximation can be computed with the average exchange charge density proposed by Slater $\underline{6}$. Another approximation, proposed by Sharp and Horton ${ }^{7}$, consists in attaining a local potential that approximates the exchange operator through a variational procedure that minimizes the energy. There are several other more elaborated methods that allow us to obtain local exchange potentials $\underline{10}-13$. However, these potentials are rather difficult to put in a simple and smooth analytical expression, such as Eq.(7).

Due to the fact that the Hartree-Fock method does not take into account the correlations, our procedure allowed us to obtain in a rather direct way "exact" local orbital-dependent exchange potentials,

$$
V_{n l}^{\mathrm{DIMx}}(r)=V_{n l}^{\mathrm{DIM}}(r)+\frac{Z_{N}}{r}-\int \frac{\rho^{\mathrm{HF}}\left(r^{\prime}\right)}{\left|\mathbf{r}-\mathbf{r}^{\prime}\right|} d \mathbf{r}^{\prime},
$$

where $\rho^{\mathrm{HF}}(r)$ is the total density calculated with the $u_{n l}^{\mathrm{HF}}(r)$ wavefunctions. Figure 2 shows the orbital-specific exchange potentials $V_{n l}^{\mathrm{DIMx}}(r)$ for the ground states of the four noble gases $\mathrm{He}, \mathrm{Ne}, \mathrm{Ar}$, and $\mathrm{Kr}$, calculated with the depurated inversion method DIM.

In order to discuss our results, in Fig. 2 we plotted the optimized effective potential $V_{\mathrm{OEP}}^{\mathrm{x}}(r)$ developed by Talman $\underline{9}$ (black dotted lines) for the noble gases. It is well known that the OEP method finds the potential which yields eigenfunctions that minimize the expectation value of the Hartree-Fock Hamiltonian. However, although very accurate, it always yields an energy above the HF energy. For practical applications the OEP potential works very well for 
Table 2: Total and orbital energies, mean and $\delta$ values for $\mathrm{He}, \mathrm{Ne}$, Ar and $\mathrm{Kr}$ atoms obtained from DIM effective potentials (upper rows) compared with the original Hartree-Fock values (lower rows).

\begin{tabular}{|c|c|c|c|c|c|c|}
\hline & $E$ & $n l$ & $\epsilon$ & $\langle r\rangle$ & $\langle 1 / r\rangle$ & $\delta$ \\
\hline \multirow[t]{2}{*}{$\mathrm{He}$} & -2.8616 & $1 s$ & -0.917956 & 0.927313 & 1.687251 & $8 \times 10^{-10}$ \\
\hline & -2.8617 & & -0.917956 & 0.927273 & 1.687282 & \\
\hline \multirow[t]{6}{*}{$\mathrm{Ne}$} & -128.4978 & $1 s$ & -32.772447 & 0.157491 & 9.621450 & $2 \times 10^{-6}$ \\
\hline & -128.5475 & & -32.772443 & 0.157631 & 9.618054 & \\
\hline & & $2 s$ & -1.930391 & 0.891336 & 1.640769 & $5 \times 10^{-6}$ \\
\hline & & & -1.930391 & 0.892113 & 1.632553 & \\
\hline & & $2 p$ & -0.850410 & 0.967755 & 1.430252 & $6 \times 10^{-6}$ \\
\hline & & & -0.850410 & 0.965274 & 1.435350 & \\
\hline \multirow[t]{10}{*}{$\mathrm{Ar}$} & -526.8030 & $1 s$ & -118.610352 & 0.086015 & 17.561606 & $2 \times 10^{-6}$ \\
\hline & -526.8175 & & -118.610350 & 0.086104 & 17.553229 & \\
\hline & & $2 s$ & -12.322153 & 0.411857 & 3.562264 & $2 \times 10^{-6}$ \\
\hline & & & -12.322153 & 0.412280 & 3.555317 & \\
\hline & & $2 p$ & -9.571466 & 0.375269 & 3.449283 & $9 \times 10^{-7}$ \\
\hline & & & -9.571466 & 0.375330 & 3.449989 & \\
\hline & & $3 s$ & -1.277353 & 1.426944 & 0.967005 & $9 \times 10^{-5}$ \\
\hline & & & -1.277353 & 1.422172 & 0.961985 & \\
\hline & & $3 p$ & -0.591017 & 1.668648 & 0.817928 & $5 \times 10^{-5}$ \\
\hline & & & -0.591017 & 1.662959 & 0.814074 & \\
\hline \multirow[t]{16}{*}{$\mathrm{Kr}$} & -2752.5365 & $1 s$ & -520.165467 & 0.042441 & 35.483699 & $5 \times 10^{-7}$ \\
\hline & -2752.0549 & & -520.165468 & 0.042441 & 35.498152 & \\
\hline & & $2 s$ & -69.903081 & 0.187181 & 7.924967 & $2 \times 10^{-6}$ \\
\hline & & & -69.903082 & 0.187256 & 7.918830 & \\
\hline & & $2 p$ & -63.009784 & 0.161695 & 7.874355 & $3 \times 10^{-6}$ \\
\hline & & & -63.009785 & 0.161876 & 7.868429 & \\
\hline & & $3 s$ & -10.849466 & 0.537875 & 2.644610 & $2 \times 10^{-6}$ \\
\hline & & & -10.849466 & 0.537802 & 2.637556 & \\
\hline & & $3 p$ & -8.331501 & 0.542133 & 2.530080 & $2 \times 10^{-6}$ \\
\hline & & & -8.331501 & 0.542627 & 2.522775 & \\
\hline & & $3 d$ & -3.825234 & 0.550922 & 2.276713 & $4 \times 10^{-6}$ \\
\hline & & & -3.825234 & 0.550880 & 2.276940 & \\
\hline & & $4 s$ & -1.152935 & 1.630081 & 0.808453 & $1 \times 10^{-4}$ \\
\hline & & & -1.152935 & 1.629391 & 0.804188 & \\
\hline & & $4 p$ & -0.524186 & 1.950193 & 0.675555 & $3 \times 10^{-5}$ \\
\hline & & & -0.524187 & 1.951611 & 0.669219 & \\
\hline
\end{tabular}



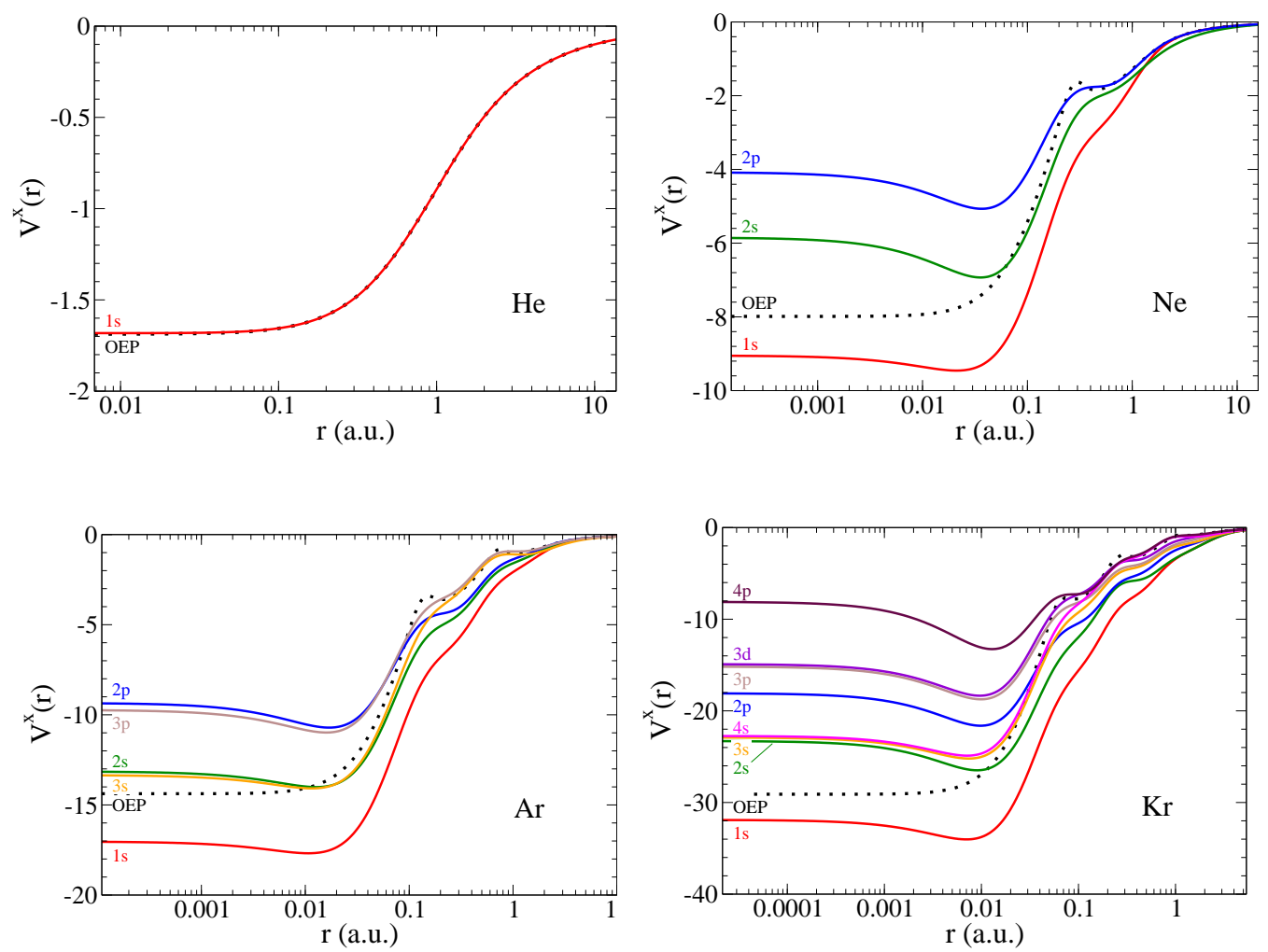

Figure 2: (color online). Orbital-specific exchange potentials $V_{n l}^{\mathrm{DIMx}}(r)$ and $V_{\mathrm{OEP}}^{\mathrm{x}}$, for the ground state of $\mathrm{He}, \mathrm{Ne}, \mathrm{Ar}$ and $\mathrm{Kr}$. 
Table 3: Orbital and total exchange energies for $\mathrm{He}, \mathrm{Ne}, \mathrm{Ar}$, and $\mathrm{Kr}$.

\begin{tabular}{c|cc|cccc|cc}
\hline & $l$ & $n$ & 1 & 2 & 3 & 4 & Total & EAHF \\
\hline $\mathrm{He}$ & 0 & -0.5129 & & & & -1.0258 & -1.026 \\
$\mathrm{Ne}$ & 0 & -3.1106 & -0.8620 & & & -12.1080 & -12.11 \\
& 1 & & -0.6938 & & & & \\
$\mathrm{Ar}$ & 0 & -5.8760 & -1.9470 & -0.5742 & & -30.1826 & -30.19 \\
& 1 & & -1.7974 & -0.4340 & & & \\
$\mathrm{Kr}$ & 0 & -12.2258 & -4.5523 & -1.9972 & -0.5275 & -93.8525 & -93.89 \\
& 1 & & -4.4305 & -1.8401 & -0.3906 & & \\
& 2 & & & -1.5280 & & & \\
\hline
\end{tabular}

the outer shell. At longer distances, all the $n l$ orbitals have a similar behavior accompanying the OEP exchange potential. We noticed that the exchange potentials of the orbitals having a common angular momentum $l$ resemble to each other (see Ar for instance). This was suggested in a work by Herman et al. $\stackrel{43}{n}$ where an $l$-averaged exchange potential for each set of electronic states was calculated as a modification of Slater's average exchange potential.

According to Eq. (10) all orbital-specific potentials should approach the same value at $r=0$, since $Z_{n l}^{\mathrm{DIM}}(r)=r V_{n l}^{\mathrm{DIM}}(r)$ approaches $Z_{N}$ regardless of $n l$ (the second and third term are the same for every orbital). However, from Fig. 2 it appears that the potentials for the different orbitals approach different values at the origin. This is a consequence of the fact that every DIM potential tends to $Z_{N}$ with different behavior, determined by their fitting parameters. In fact, for very low $r$ values $V_{n l}^{\mathrm{DIM}}(r) \approx \sum_{j} \alpha_{j} \beta_{j}-V^{\mathrm{d}}(r)$, but they all have strictly the same value at $r=0$.

As a final test for our method, we calculated the total exchange energy $E^{\mathrm{x}}$ as given by

$$
E^{\mathrm{x}}=\sum_{n l} E_{n l}^{\mathrm{x}}=\sum_{n l}\left[\frac{1}{2} \int \rho_{n l}^{\mathrm{HF}}(r) V_{n l}^{\mathrm{DIMx}}(r) d r\right]
$$

Table 3 displays the orbital exchange energy as well as the total exchange energy for He, Ne, $\mathrm{Ar}$ and Kr. The total exchange energies are compared with the exact atomic Hartree-Fock $(\mathrm{EAHF})$ values given by Becke$\stackrel{44}{ }$, with very good agreement.

\subsection{Nitrogen DIM and Exchange Potentials}

The procedure developed here is not limited to noble gases or closed shells. As an example we will apply the method to Nitrogen. The lower configuration $2 p^{3}$ of Nitrogen gives rise to three different terms: $2{ }^{4} \mathrm{~S}, 2{ }^{2} \mathrm{D}, 2{ }^{2} \mathrm{P}$. Each of them is described by a different electronic density. The fitting parameters that define the term-dependent effective charges are given in Table 4 for each of the terms. We built the corresponding DIM potentials from these effective charges. By using these potentials we solved the Schrödinger equation (Eq.(1)) for every term, obtained the solutions, the energies, and the corresponding mean values $\langle r\rangle$ and $\langle 1 / r\rangle$. The comparison between the orbitals obtained from the diagonalization of the Hamiltonian with the effective potentials and the original Hartree-Fock orbitals are shown 
Table 4: Fitting parameters for the effective charge $Z_{n l}^{\mathrm{DIM}}(r)$ for $2{ }^{4} \mathrm{~S}, 2^{2} \mathrm{D}$ and $2{ }^{2} \mathrm{P}$ terms of Nitrogen.

\begin{tabular}{ccccccc}
\hline & \multicolumn{2}{c}{$2{ }^{4} \mathrm{~S}$} & \multicolumn{2}{c}{$2{ }^{2} \mathrm{D}$} & \multicolumn{2}{c}{$2{ }^{2} \mathrm{P}$} \\
$n l$ & $\alpha$ & $\beta$ & $\alpha$ & $\beta$ & $\alpha$ & $\beta$ \\
\hline $1 s$ & 5.25634 & 1.26207 & 5.18635 & 1.22410 & 5.18635 & 1.21779 \\
& 0.743660 & 8.02844 & 0.813650 & 7.56800 & 0.813650 & 7.56740 \\
$2 s$ & 2.45281 & 3.51271 & 0.398100 & 0.239738 & 0.890660 & 0.830615 \\
& 0.833570 & 3.38654 & 1.85412 & 1.03105 & 3.66999 & 3.14946 \\
& 2.71362 & 0.894699 & 3.74778 & 2.85313 & 1.43935 & 0.740427 \\
$2 p$ & 3.64345 & 1.24069 & 4.01052 & 1.28744 & 1.89769 & 1.16557 \\
& 2.05501 & 5.35135 & 1.85517 & 5.70858 & 1.77430 & 5.68782 \\
& 0.301540 & 0.286609 & 0.134310 & 0.267987 & 2.32801 & 1.40925 \\
\hline
\end{tabular}

in Table 5. The mean values $\langle r\rangle$ obtained with the DIM effective potentials agree with the $\mathrm{HF}$ values in about $0.1 \%$, and the $\langle 1 / r\rangle$ mean values agree in about $0.2 \%$. The calculated total energies $E^{\text {DIM }}$ for each term of the Nitrogen atom using Eq. (11) are presented in Table 5. The agreement between the DIM total energies and the original HF total energies is excellent, of about $0.04 \%$. Figure 3 shows the $n l$-orbital exchange potentials for the $2{ }^{4} \mathrm{~S}$, $2^{2} \mathrm{D}$ and $2^{2} \mathrm{P}$ terms, calculated with the depurated inversion method. Again, to compare our results, the exchange potential given by Talman $\underline{9}$ (OEP) is presented in the figures in light grey. Figure 3(a) illustrates the exchange potential for the $1 s$ orbitals for the different terms, showing an overall similarity. The OEP potential behaves like the $V_{1 s}^{\mathrm{xDIM}}(r)$ only at short and large distances. Figure 3(b) shows the exchange potentials for the $2 s$ orbitals. In this case, noticeable differences between the term-potentials arise at low values of $r$. For $r$ higher than 0.5 a.u., all the term-potentials become indistinguishable and agree perfectly with the OEP potential. A pecularity observed in the figure is that the OEP potential agrees very well with the $V_{2 s}^{\mathrm{xDIM}}(r)$ for the $2^{2} D$ term. Figure 3 (c) displays the $2 p$ exchange potentials, which behave similarly for all the terms. However, since the OEP potential is the same for all the orbitals and terms, it disagrees completely with the $V_{2 p}^{\mathrm{x}}(r)$ at short distances.

Table 6 presents the total exchange energy and the $n l$-exchange energies of the $2{ }^{4} \mathrm{~S}, 2{ }^{2} \mathrm{D}$ and $2^{2} \mathrm{P}$ terms. The $1 s$-exchange energy for all the terms are the same, as expected for a closed-shell orbital. Similarly, the $2 s$-exchange energy varies slightly, with a difference of $0.08 \%$. However, this is not the case for the $2 p$-exchange energy, which varies significantly, having discrepancies of about $18 \%$ between the different terms. The total exchange energy computed with Eq. (11) for the terms are compared with the exact atomic Hartree-Fock (EAHF) exchange energy, with an agreement of about $0.1 \%$.

\section{CONCLUSIONS}

A crucial requirement of the density functional method is the accurate representation of the exchange functional. On the other hand, the atomic collision community needs accurate oneelectron potentials in order to generate the bound and continuum states on the same footing for further calculations of collisional processes. These potentials need to be worked out for 
Table 5: Total and orbital energy and mean values for the $2{ }^{4} \mathrm{~S}, 2^{2} \mathrm{D}$ and $2{ }^{2} \mathrm{P}$ terms of $\mathrm{N}$ obtained from the DIM effective potentials (upper rows) compared with the Hartree-Fock values (lower rows).

\begin{tabular}{|c|c|c|c|c|c|}
\hline & $E$ & $n l$ & $\epsilon$ & $\langle r\rangle$ & $\langle 1 / r\rangle$ \\
\hline \multirow[t]{6}{*}{$2{ }^{4} \mathrm{~S}$} & -54.37617 & $1 s$ & -15.62906 & 0.22830 & 6.64863 \\
\hline & -54.40093 & & -15.62906 & 0.22830 & 6.65324 \\
\hline & & $2 s$ & -0.94532 & 1.33448 & 1.08037 \\
\hline & & & -0.94532 & 1.33228 & 1.07818 \\
\hline & & $2 p$ & -0.56759 & 1.41268 & 0.95498 \\
\hline & & & -0.56759 & 1.40963 & 0.95769 \\
\hline \multirow[t]{6}{*}{$2^{2} \mathrm{D}$} & -54.27557 & $1 s$ & -15.66639 & 0.22829 & 6.64929 \\
\hline & -54.29617 & & -15.66639 & 0.22826 & 6.65388 \\
\hline & & $2 s$ & -0.96367 & 1.32917 & 1.08644 \\
\hline & & & -0.96367 & 1.32632 & 1.08318 \\
\hline & & $2 p$ & -0.50866 & 1.44878 & 0.93882 \\
\hline & & & -0.50866 & 1.44662 & 0.94208 \\
\hline \multirow[t]{6}{*}{$2^{2} \mathrm{P}$} & -54.20856 & $1 s$ & -15.69160 & 0.22824 & 6.65036 \\
\hline & -54.22810 & & -15.69160 & 0.22824 & 6.65430 \\
\hline & & $2 s$ & -0.97634 & 1.32562 & 1.08712 \\
\hline & & & -0.97634 & 1.32232 & 1.08656 \\
\hline & & $2 p$ & -0.47130 & 1.47176 & 0.92982 \\
\hline & & & -0.47130 & 1.47301 & 0.93155 \\
\hline
\end{tabular}

Table 6: Orbital and total exchange energies for $2{ }^{4} \mathrm{~S}, 2{ }^{2} \mathrm{D}$ and $2{ }^{2} \mathrm{P}$ terms of Nitrogen.

\begin{tabular}{cccccc}
\hline & $1 \mathrm{~s}$ & $2 \mathrm{~s}$ & $2 \mathrm{p}$ & Total & EAHF \\
\hline $2{ }^{4} \mathrm{~S}$ & -2.1175 & -0.4776 & -0.4711 & -6.6034 & -6.596 \\
$2{ }^{2} \mathrm{D}$ & -2.1175 & -0.4777 & -0.4262 & -6.4688 & \\
$2{ }^{2} \mathrm{P}$ & -2.1175 & -0.4780 & -0.3973 & -6.3827 & \\
\hline
\end{tabular}



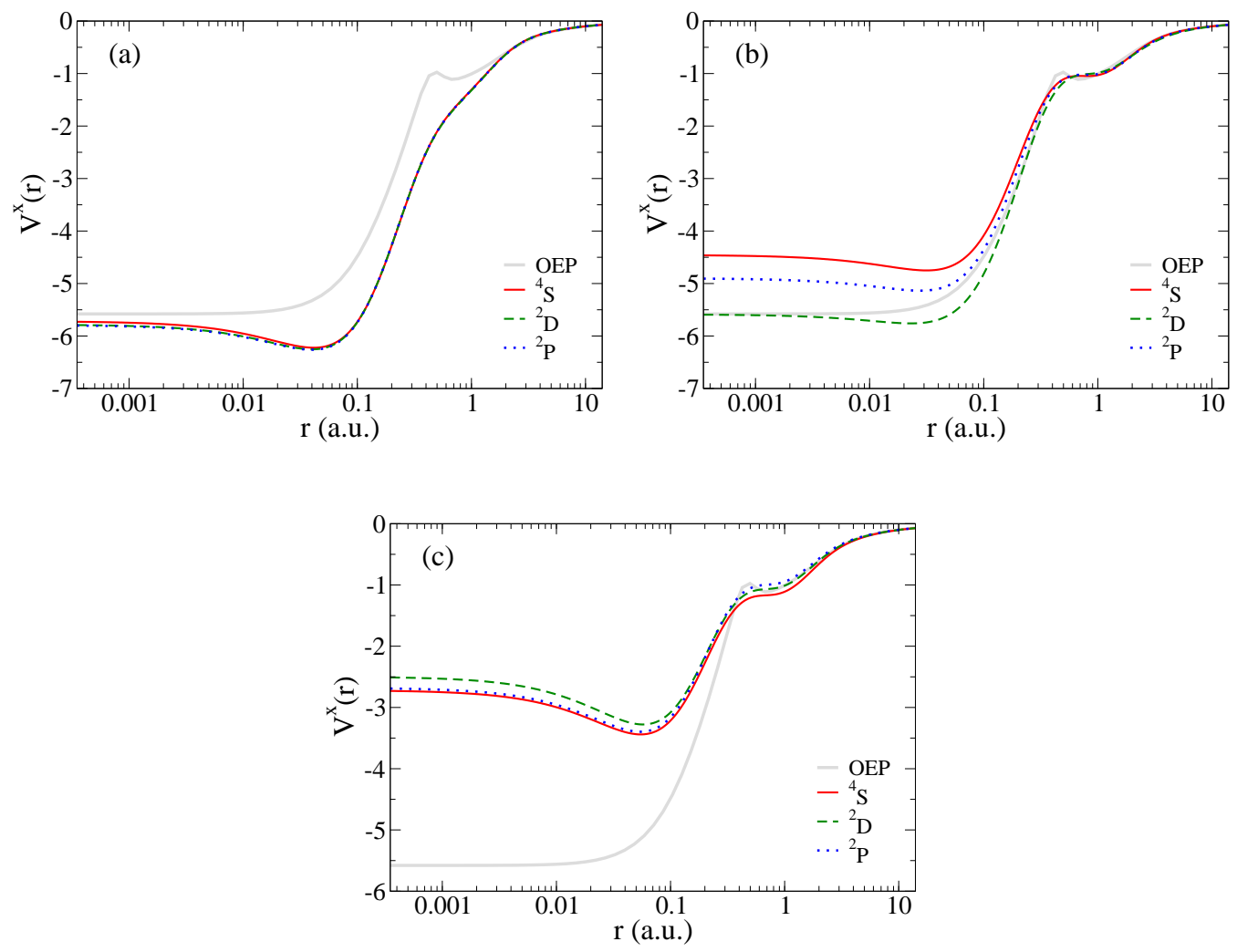

Figure 3: (color online). DIM exchange potential $V_{n l}^{\mathrm{DIMx}}(r)$ for the (a) $1 s$, (b) $2 s$ and (c) $2 p$ orbitals, for the $2{ }^{4} \mathrm{~S}, 2{ }^{2} \mathrm{D}$ and $2{ }^{2} \mathrm{P}$ terms of Nitrogen. 
any $n l$-specific orbital, a feature that in general is not present in the chemistry community functionals. In the present work we devised and implemented a depurated inversion method, which allows to obtain the intended potentials through a very simple analytical expression of the effective charges. The method consists in the inversion of a Kohn-Sham equation, in which the KS orbitals have been replaced by the Hartree-Fock orbitals. By means of diagonalization we have achieved accurate wavefunctions having almost perfect agreement with the original Hartree-Fock wave functions. The quality of the potentials obtained by the present method is remarkably good. We applied the developed methodology to the calculation of the ground state orbitals of noble gases and the Nitrogen atom. It is worth mentioning that the same technique can be used for any other level, i.e., it is not limited to the ground state.

\section{ACKNOWLEDGMENTS}

This work was supported by grants of CONICET, ANPCyT, and UBACyT, of Argentina.

\section{References}

[1] P. Hohenberg, W. Kohn, Phys. Rev. 1964, 136, B864

[2] W. Kohn, L. J. Sham, Phys. Rev. 1965, 140, A1133

[3] A. D. Becke, J. Chem. Phys. 2014, 140, 18A301.

[4] R. J. Bartlett, Mol. Phys. 2010, 108, 3299-3311.

[5] P. Verma, R. J. Bartlett, J. Chem. Phys. 2012, 137, 134102.

[6] J. C. Slater, Phys. Rev. 1981, 81, 385.

[7] R. T. Sharp, G. K. Horton, Phys. Rev. 1953, 90, 317.

[8] J. D. Talman, W. F. Shadwick, Phys. Rev. A 1976, 14, 36-40.

[9] J. D. Talman, Comput. Phys. Commun. 1989, 54, 85-94.

[10] J. B. Krieger, Y. Li, G. J. Iafrate, Phys. Rev. A 1992, 45, 101-126.

[11] A. Görling, Phys. Rev. A 1992, 46, 3753-3757.

[12] W. Yang, Q. Wu, Phys. Rev. Lett. 2002, 89, 143002.

[13] V. N. Staroverov, G. E. Scuseria and E. R. Davidson, J. Chem. Phys. 2006, 124, 11103.

[14] I. G. Ryabinkin, A. A. Kananenka, and V. N. Staroverov, Phys. Rev. Lett. 2013, 111, 013001.

[15] ABINIT, WWW.abinit.org 
[16] Vanderbilt Ultra-Soft Pseudopotential, www.physics.rutgers.edu/ dhv/uspp/

[17] A. P. Gaiduk, I. G. Ryabinkin, V. N. Staroverov, J. Chem. Theory Comput. 2013, 9, 3959.

[18] Q. Wu, W. Yang, J. Chem. Phys. 2003, 118, 2498.

[19] I. G. Ryabinkin, S. V. Kohut, V. N. Staroverov, Phys. Rev. Lett. 2015, 115, 083001.

[20] M. E. Mura, P. J. Knowles, C. A. Reynolds, J. Chem. Phys. 1997, 106, 9659.

[21] C. J. Umrigar, X. Gonze, Phys. Rev. A 1994, 50, 3827.

[22] O. V. Gritsenko, E. J. Baerends, Theor. Chem. Acc. 1997, 96, 44.

[23] C. Filippi, C. J. Umrigar, M. Taut, J. Chem. Phys. 1994, 100, 1290.

[24] P. de Silva, T. A. Wesolowski, Phys. Rev. A 2012, 85, 032518.

[25] P. R. T. Schipper, O. V. Gritsenko, E. J. Baerends, Theor. Chem. Acc. 1997, 98, 16.

[26] A. A. Kananenka, S. V. Kohut, A. P. Gaiduk, and I. G. Ryabinkin, J. Chem. Phys. 2013, 139, 074112.

[27] C. R. Jacob, J. Chem. Phys. 2011, 135, 244102.

[28] P. R. Hilton, S. Nordholm, N. S. Hush, J. Chem. Phys. 1997, 67, 5213.

[29] S. Süzer, P. R. Hilton, N. S. Hush, S. Nordholm, J. Elect. Spec. Rel. Phen. 1977, 12, 357.

[30] P. R. Hilton, S. Nordholm, N. S. Hush, Chem. Phys. Lett. 1979, 64, 515.

[31] P. R. Hilton, S. Nordholm, N. S. Hush, J. Elect. Spec. Rel. Phen. 1980, 18, 101.

[32] Ž. Crljen, G. Wendin, Phys. Rev. A 1987, 35, 1571.

[33] R. M. Sternheimer, Phys. Rev. 1954, 96, 951.

[34] A. Dalgarno, D. Parkinson, Proc. R. Soc. Lond. A 1959, 250, 422.

[35] V. Barone, J. Chem. Phys. 1994, 101, 6834.

[36] M. Kaupp, ; A. V. Arbuznikov, A. Heßelmann, A. Görling, J. Chem. Phys. 2010, 132 184107.

[37] C. Froese Fischer, T. Brage, P. Jönsson, Computational Atomic Structure, an MCHF approach, Institute of Physics Publishing, 1997.

[38] W. R. Johnson, Atomic Structure Theory: Lectures on Atomic Physics, Springer, 2007.

[39] M. J. G. Peach, D. G. J. Griffiths, D. J. Tozer, J. Chem. Phys. 2012, 136, 144101. 
[40] B. J. Albright, K. Bartschat, P. R. Flicek, J. Phys. B 1993, 26, 337.

[41] K. Bartschat, Computational Atomic Physics, Springer-Verlag, 1996; Chapter II.

[42] K. Bartschat, I. Bray, J. Phys. B 1996, 29, 271.

[43] F. Herman, J. Callaway and F. S. Acton, Phys. Rev. 1954, 95, 371.

[44] A. D. Becke, Phys. Rev. A 1988, 38, 3098. 